\title{
The Effect of Numerical Techniques on Differential Equation Based Chaotic Generators
}

\author{
M. Affan $\operatorname{Zidan}^{1}$, A. G. Radwan ${ }^{1,2}$ and K. N. Salama ${ }^{1}$ \\ ${ }^{1}$ Electrical Engineering Program, King Abdullah University of Science and Technology (KAUST) \\ Thuwal, Kingdom of Saudi Arabia 23955-6900 \\ Email: \{mohammed.zidan, ahmed.radwan, khaled.salama\}@kaust.edu.sa \\ ${ }^{2}$ Department of Engineering Mathematics, Faculty of Engineering, Cairo University, Egypt
}

\begin{abstract}
In this paper, we study the effect of the numerical solution accuracy on the digital implementation of differential chaos generators. Four systems are built on a Xilinx Virtex 4 FPGA using Euler, mid-point, and Runge-Kutta fourth order techniques. The twelve implementations are compared based on the FPGA used area, maximum throughput, maximum Lyapunov exponent, and autocorrelation confidence region. Based on circuit performance and the chaotic response of the different implementations, it was found that less complicated numerical solution has better chaotic response and higher throughput.
\end{abstract}

\section{INTRODUCTION}

Chaotic circuits find many applications in cryptography [1], [2] and random number generation [3]. Moreover, chaos is used in digital communication systems as chaos shift keying (CSK) [4]. Chaos generators can be implemented using operational amplifiers [5], discrete MOS transistors [6], and fully integrated [7].

The analog chaotic generators are sensitive to the running conditions, process variations, and the operating temperature. Also, tolerances in analog realization degrade the quality of the chaos output [8]. Moreover, the initial conditions cannot be set precisely in the analog generator, and the circuit capacitors require a huge on-chip area. In addition, using analog generators in digital systems requires digitization of the output. On the other hand, the digital chaotic systems overcome these problems, and can be easily synchronized in communications and encryption systems.

Digital chaos is mostly based on logistic maps [9]. However Differential equation based chaos provides more nonlinearity and is of higher dimensional order compared to the logistic maps. The increased complexity enables building more secured chaos based systems.

Recently an all digital implementation of differential equation based systems has been introduced [3], [10]. Also a differential equation based multi-scroll system is presented in [11]. The differential equation based chaos was implemented by realizing its numerical solution. In this paper we study different numerical solutions (Euler, mid-point, and Rung-Kutta fourth) and compare their performance. We also show that the procedure is universal by studying four different systems with different nonlinearities and comparing their results. The four used systems are given in Table I.

All the systems implemented on a Xilinx Virtex 4 FPGA, using three different numerical techniques. The twelve differ- ent implementations are compared based on their areas, the maximum throughput. The chaotic behavior is also compared based on the Maximum Lyapunov Exponent (MLE) and the autocorrelation confidence region. We show that the Euler technique provides the best performance compared to midpoint and Runge-Kutta $4^{\text {th }}$-order, based of the mentioned comparison technique. Also, The midpoint express better response with respect to Runge-Kutta $4^{\text {th }}$-order.

\section{Digital Implementation AND Numerical TECHNIQUES}

Digital generators for differential equation based chaos are implemented by realizing the numerical solution of the system equations. The state variables of the system are realized as registers, and the numerical solution of each variable are implemented as combinational block, as shown in Fig. 1.

Many circuit optimizations can be introduced to improve the performance of the combinational part. One of these optimizations is the elimination of constant multiplications with the aid of suitable selection of the system parameters to be in the powers of two. What make this optimization possible is the fact that chaos behavior are expressed for interval of parameters, not a single value. Another important optimization is using fixed point representation scheme for realizing chaotic circuit. This is based on that the outputs of the chaotic generator are within bounded intervals.

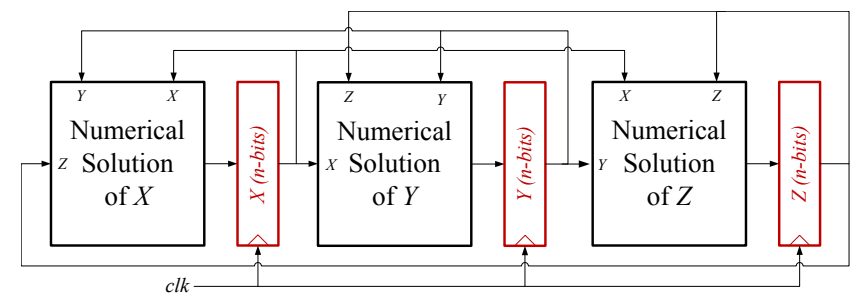

Fig. 1. The general block diagram of the digital implementation of 3D differential based chaos generator.

One of the main design point of the digital chaos generator is the selection of numerical solution. The adopted numerical technique will affect the chaotic response of the realization. We select three known numerical solution, with different complexities and accuracies, to study their effect on the response 
TABLE I

THE CHAOTIC SYSTEMS USED WITHIN THE COMPARISONS.

\begin{tabular}{c|c|c|c|c}
\hline & $\begin{array}{c}\text { System 1 } \\
\text { Lorenz [12] }\end{array}$ & System 2 & System 3 & $\begin{array}{c}\text { System 4 } \\
\text { Elwakil [5] }\end{array}$ \\
\hline \multirow{3}{*}{ Equations } & $\dot{X}=\alpha(Y-X)$ & $\dot{X}=a(Y-X)$ & $\dot{X}=a(Y-X)$ & $\dot{X}=Y$ \\
& $\dot{Y}=(\rho-Z) X-Y$ & $\dot{Y}=\operatorname{sign}(x)(b-Z)+c y$ & $\dot{Y}=\operatorname{sign}(x)(b-Z)$ & $\dot{Y}=Z$ \\
& $\dot{Z}=X Y-\beta Z$ & $\dot{Z}=|y|-d Z$ & $\dot{Z}=|x|-c Z$ & $\dot{Z}=-Z-Y B(Y)-X$ \\
\hline Nonlinearity & Multiplication & Sign, Modules & Sign, Modules & Piecewise \\
\hline
\end{tabular}

of the circuit. Assume the following differential equation:

$$
\dot{A}=F_{A}(A, B, \ldots)
$$

This equation is solved using the following techniques:

\section{Euler Method:}

It is a simple technique which required one calculation per numerical iteration, in return it produces less accurate results compared with other more complex technique. Euler method is defined as,

$$
A_{i+1}=A_{i}+h \cdot F_{A}\left(A_{i}, B_{i}, \ldots\right)
$$

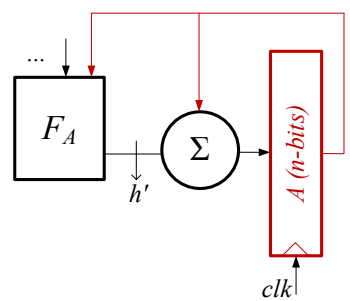

Fig. 2. Block diagram of the digital circuit used to evaluate numerical solution using Euler technique. $h^{\prime}=\log _{2}(h)$ represents shift in wires for implementing the arithmetic shift.

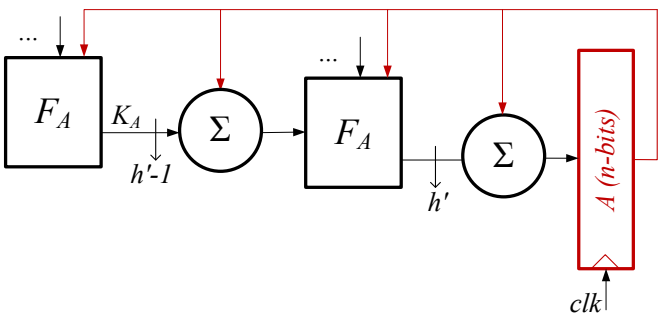

Fig. 3. Block diagram of the digital circuit used to evaluate numerical solution using midpoint technique. $h^{\prime}=\log _{2}(h)$ represents shift in wires for implementing the arithmetic shift. where $A_{i}$ and $A_{i+1}$ are the solutions of the variable $A$ at steps $i$ and $i+1$ respectively, and $h$ is the time step. Fig. 2 shows the the schematic of the Euler numerical solution.

Midpoint Method: This method is defined as,

$$
\begin{gathered}
K_{A}=F_{A}\left(A_{i}, B_{i}, \ldots\right) \\
A_{i+1}=A_{i}+h \cdot F_{A}\left(A_{i}+0.5 h K_{A}, B_{i}+0.5 h K_{B}, \ldots\right)
\end{gathered}
$$

Compared to Euler, this technique produces more accurate numerical iterations. On the other hand, this technique requires more hardware, and longer calculation path as shown in Fig. 3

Runge-Kutta $4^{\text {th }}$-order: This method have the longest calculation path and requires large area, but it produces the most accurate numerical approximation compared to the previous technicians. Runge-Kutta $4^{\text {th }}$-order is defined as,

$$
\begin{gathered}
K_{A 1}=F_{A}\left(A_{i}, B_{i}, \ldots\right) \\
K_{A 2}=F_{A}\left(A_{i}+0.5 h K_{A 1}, B_{i}+0.5 h K_{B 1}, \ldots\right) \\
K_{A 3}=F_{A}\left(A_{i}+0.5 h K_{A 2}, B_{i}+0.5 h K_{B 2}, \ldots\right) \\
K_{A 4}=F_{A}\left(A_{i}+h K_{A 3}, B_{i}+h K_{B 3}, \ldots\right) \\
A_{i+1}=A_{i}+h\left(K_{1}+2 K_{2}+2 K_{3}+K_{4}\right) / 6
\end{gathered}
$$

Fig. 4 shows that evaluating the solution using the RungeKutta $4^{\text {th }}$-order method which have the longest pipeline.

\section{EXPERIMENTAL RESULTS}

The twelve circuits implemented using Verilog VHDL on Xilinx Virtex ${ }^{\circledR} 4$ XC4VSX35 FPGA. We used 32 bits fixedpoint scheme for representing the numbers. GNU iVerilog software was used for circuit simulation, and Xilinx ISE ${ }^{\circledR} 11$, with optimized for time (speed) option selected, was used for synthesizing the circuit and calculating the used area and maximum frequency. The attractors and the time diagrams are captured using TekTronix ${ }^{\mathrm{TM}}$ MSO 414 oscilloscope.

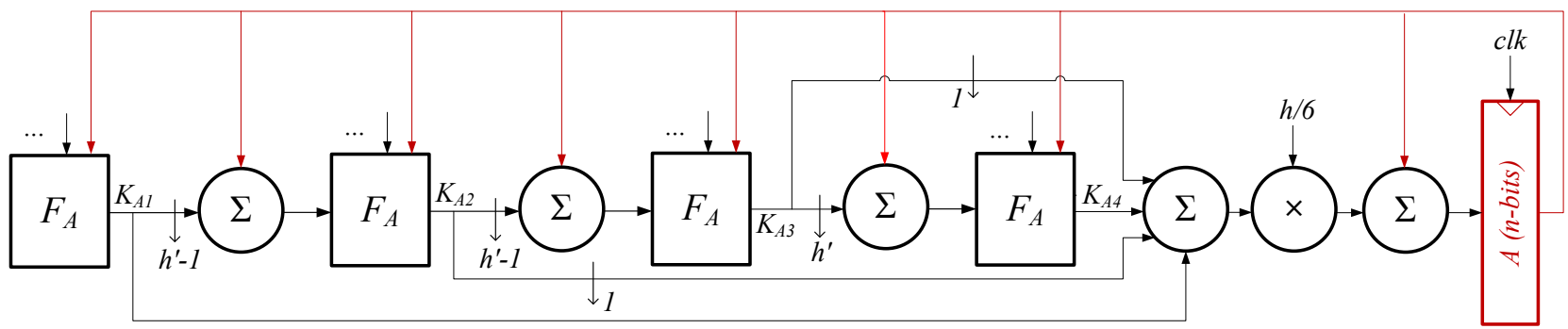

Fig. 4. Block diagram of the digital circuit used to evaluate numerical solution using Runge-Kutta $4^{t h}$-order technique. $h^{\prime}=\log _{2}(h)$ represents shift in wires for implementing the arithmetic shift. 
TABLE II

DETAILED COMPARISON FOR THE FOUR SYSTEMS. THE CIRCUIT DATA IS GIVEN IMPLEMENTATION ON XILINX VIRTEX $4{ }^{\circledR}$ XC4VSX35 FPGA.

\begin{tabular}{|c|c|c|c|c|c|c|c|c|c|}
\hline System & $\begin{array}{l}\text { Numerical } \\
\text { Method }\end{array}$ & $\begin{array}{c}\text { Slices } \\
(\mathbf{1 5 , 3 6 0 )}\end{array}$ & $\begin{array}{l}\text { Slice FF } \\
(\mathbf{3 0 , 7 2 0})\end{array}$ & $\begin{array}{l}\text { LUTs } \\
(\mathbf{3 0 , 7 2 0})\end{array}$ & $\begin{array}{l}\text { DSPs } \\
(192)\end{array}$ & $\begin{array}{l}\text { Freq. } \\
\text { (MHz) }\end{array}$ & $\begin{array}{c}\text { Throughput } \\
\text { (Gb/s) }\end{array}$ & $\begin{array}{c}\text { Autocorrelation } \\
\text { (95\% Conf. Range) }\end{array}$ & $\begin{array}{c}\text { MLE } \\
\text { (250k iter.) }\end{array}$ \\
\hline & Euler & 145 & 96 & 287 & 8 & 53.53 & 1.71 & 23 & 1.667 \\
\hline 1 & MP & 222 & 96 & 440 & 16 & 28.00 & 0.90 & 36 & 1.179 \\
\hline Lorenz [12] & RK $4 \mathrm{O}$ & 658 & 97 & 1,216 & 32 & 13.17 & 0.42 & 38 & 1.167 \\
\hline & Euler & 144 & 97 & 284 & 0 & 122.01 & 3.90 & 183 & 0.299 \\
\hline 2 & MP & 286 & 96 & 568 & 0 & 72.01 & 2.30 & 188 & 0.267 \\
\hline Chen [13] & RK 40 & 860 & 98 & 1,611 & 0 & 28.02 & 0.90 & 202 & 0.236 \\
\hline & Euler & 137 & 97 & 265 & 0 & 126.73 & 4.06 & 1,137 & 0.027 \\
\hline 3 & MP & 295 & 96 & 547 & 0 & 77.56 & 2.48 & 1,139 & 0.022 \\
\hline Elwakil [5] & RK 40 & 916 & 96 & 1,650 & 0 & 33.51 & 1.07 & 1,219 & 0.020 \\
\hline & Euler & 109 & 97 & 215 & 0 & 129.52 & 4.14 & 2,982 & 0.159 \\
\hline 4 & MP & 195 & 96 & 385 & 0 & 62.95 & 2.01 & 6,540 & 0.146 \\
\hline Elwakil [5] & RK 40 & 597 & 97 & 1,140 & 0 & 29.73 & 0.95 & 117,262 & 0.106 \\
\hline
\end{tabular}

The detailed FPGA utilization and maximum frequency for the different implementations are given in Table II. In general, system 1 requires more area and express lower throughput because it uses multiplication as nonlinear element. As shown in Fig 2, 3 and 4, the main block $F_{A}$ is serially repeated two and four times for the midpoint and Runge-Kutta $4^{\text {th }}$-order methods compared to one appearance in the Euler technique. This repetition will have an impact on the area and delay with almost the same factor. Based on the number of FPGA slices used, Euler and midpoint implantations require $15-22 \%$ and $32-34 \%$ of the area required by Runge-Kutta $4^{t h}$-order respectively. The same approximate factor appears for the number of DSPs used in system 1, which is the only system that uses this blocks due to its multiplication nonlinear element. In the same direction, Euler and midpoint implantations expresses $3.8-4.4 \mathrm{x}$ and 2.1-2.5x higher throughput compared to RungeKutta $4^{t h}$-order respectively. The number of the used registers is almost independent of the numerical method.

Fig. 5 shows the oscilloscope snapshots for the $X-Z$ projection of the different implementations attractors. It appear from the figure that the used numerical technique did not

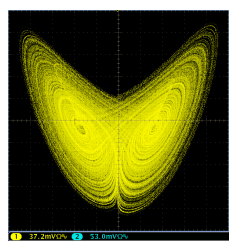

(a)

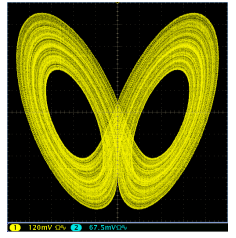

(g)

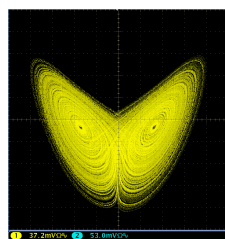

(b)

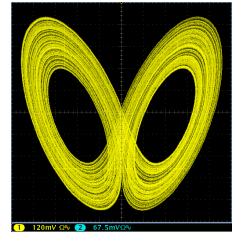

(h)

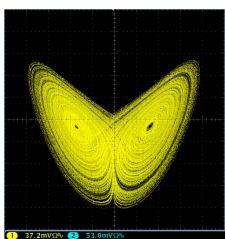

(c)

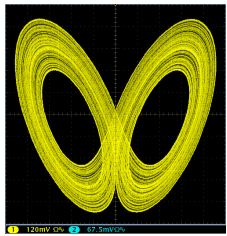

(i)

affect the pattern of the attractor. In some cases, Euler circuit produces more spread attractor compared to midpoint and Runge-Kutta $4^{\text {th }}$-order, as shown in the case of system 1 in Fig. 5(a,b,c). In the time domain response and under the same initial conditions, the circuit behavior are totally changed for the three different techniques. Fig. 6 shows that the circuit time waveform of system 1, where the Euler output jumps between scrolls more than midpoint which is more than Runge-Kutta $4^{t h}$-order. This property is useful for the chaos based random number generators that uses the sign of the output signal as a source of randomness.

\section{Chaotic Response}

The chaotic response of the different implementations is compared using the MLE, autocorrelation 95\% confidence region, and some statistical measurement. MLE is a sufficient measurement for chaotic behavior. Table II shows the calculated MLE for the different implementations using the software provided in [14]. Based on the calculated values of all systems, Euler implementation have better chaotic response than midpoint, which is also better than Runge-Kutta $4^{t h}$ order. Euler and midpoint implantations expresses $27-50 \%$ and

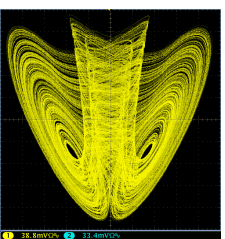

(d)

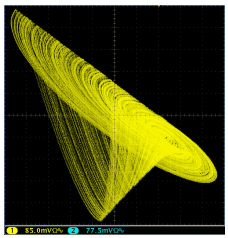

(j)

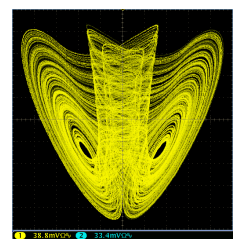

(e)

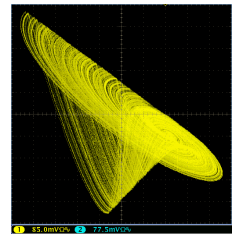

(k)

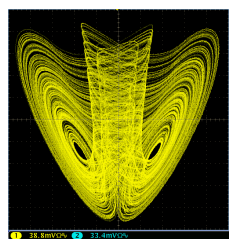

(f)

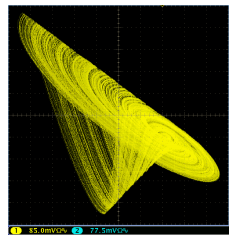

(1)

Fig. 5. Oscilloscope snapshots of the attractors projection on the $X-Z$ plane for different implementations, (a-c) system 1 , (d-f) system 2 , (g-i) system 3 and (j-1) system 4. The attractors in $(\mathrm{a}, \mathrm{d}, \mathrm{g}, \mathrm{j}),(\mathrm{b}, \mathrm{e}, \mathrm{h}, \mathrm{k})$, and $(\mathrm{c}, \mathrm{f}, \mathrm{i}, \mathrm{l})$ are for the Euler, midpoint, and Runge-Kutta 4th-order implementations, respectively. 


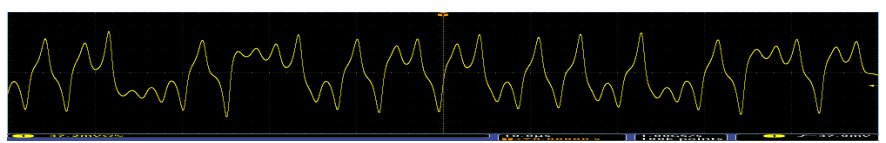

(a) Euler

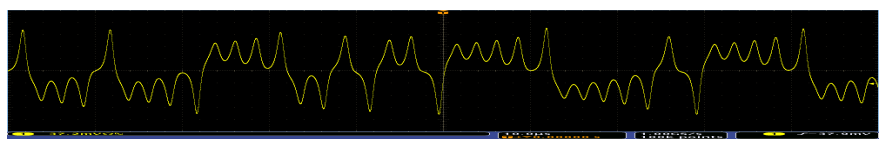

(b) Midpoint

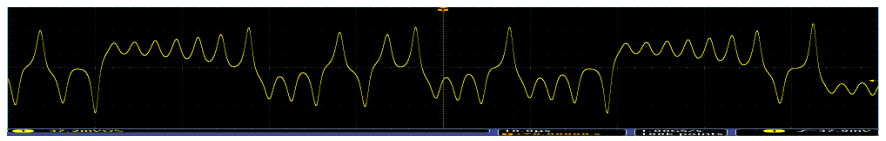

(c) Runge-Kutta $4^{\text {th }}$ order

Fig. 6. Oscilloscope snapshots of the circuit output of system 1 for the same time span and circuit frequency for the different implementations.

$1-37 \%$ higher MLE compared to Runge-Kutta $4^{\text {th }}$-order respectively. Fig. 7 shows the MLE of system 1 implementations versus the number of iterations, for the three implementations.

Another technique used to compare the output is its autocorrelation. The less correlation between the successive outputs expresses better random properties of the system. The $95 \%$ confidence region of the autocorrelation is used to compare the different techniques. The confidence region is defined by the largest lag after which the correlation value did not go out of the region bounds. The lower lag value means less correlation. Table II shows lag value for the $95 \%$ confidence region for the variable $X$ for the different implementations. In all of the cases the correlation response of Euler implementation is better chaotic response than midpoint, which is again better than Runge-Kutta $4^{t h}$-order. The autocorrelation is calculated for data sample of 900k. Fig. 8 shows the autocorrelation of the variable $X$ of system 4 different implementations.

From the previous comparison it is found that Euler implementations express better chaotic response than midpoint, which in turn is better than Runge-Kutta $4^{\text {th }}$-orde, because the numerical solution error adds an extra nonlinearity to the chaotic system.

\section{CONCLUSION}

In this paper we studied the effect of the numerical solution techniques on the performance of the digital differential based chaotic systems. Four different system are implemented on FPGA using three different numerical techniques. By comparing the four systems, It was found that Euler technique have a

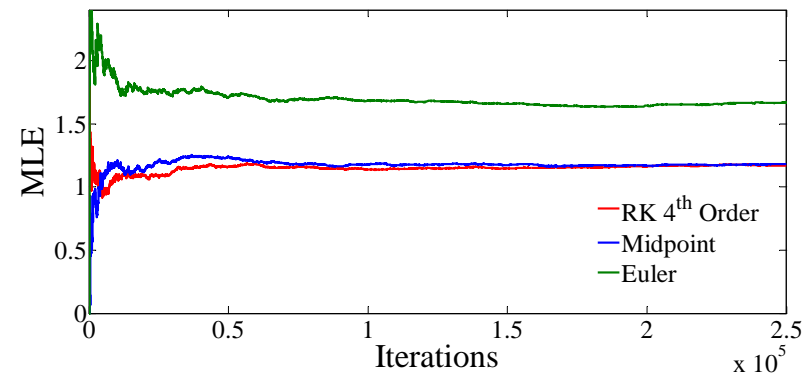

Fig. 7. The MLE iterations for the different implementations of system 1 .

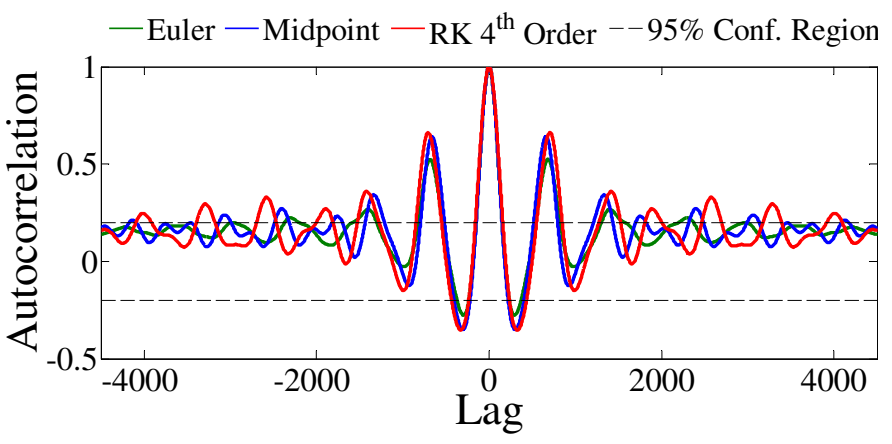

Fig. 8. Autocorrelation for the variable $X$ of system 4, for different lag scans. The dashed lines bounded the $95 \%$ confidence region.

better response than the midpoint, and the midpoint is better than the Runge-Kutta $4^{t h}$-order. The systems are compared based on the required area, maximum throughput, maximum Lyapunov exponent, and autocorrelation confidence region.

\section{REFERENCES}

[1] S. Mazloom and A. M. Eftekhari-Moghadam, "Color image encryption based on coupled nonlinear chaotic map," Chaos, Solitons \& Fractals, vol. 42, no. 3, pp. 1745-1754, 2009.

[2] M. Barakat, A. G. Radwan, and K. N. Salama, "Hardware realization of chaos based block cipher for image encryption," in IEEE International Conference on Microelectronics (ICM'11), December 2011.

[3] M. A. Zidan, A. G. Radwan, and K. N. Salama, "Random number generation based on digital differential chaos," in IEEE International Midwest Symposium on Circuits and Systems (MWSCAS'11), August 2011.

[4] Z. Galias and G. Maggio, "Quadrature chaos-shift keying: theory and performance analysis," IEEE Transactions on Circuits and Systems I: Fundamental Theory and Applications, vol. 48, no. 12, pp. 1510-1519, 2001.

[5] A. Elwakil and M. Kennedy, "Construction of classes of circuitindependent chaotic oscillators using passive-only nonlinear devices," IEEE Transactions on Circuits and Systems I: Fundamental Theory and Applications, vol. 48, no. 3, pp. 289-307, 2001.

[6] A. G. Radwan, A. M. Solman, and A. El-Sedeek, "MOS realization of the modified Lorenz chaotic system," Chaos, Solitons and Fractals, vol. 21, p. 553561, 2004.

[7] A. S. Elwakil, K. N. Salama, and M. P. Kennedy, "An equation for generating Chaos and its monolithic implementation," International Journal of Bifurcation and Chaos, vol. 12, no. 12, pp. 2885-2895, 2002.

[8] T. Addabbo, M. Alioto, A. Fort, S. Rocchi, and V. Vignoli, "A feedback strategy to improve the entropy of a chaos-based random bit generator," IEEE Transactions on Circuits and Systems I: Regular Papers, vol. 53, no. 2 , pp. 326 - 337, feb. 2006.

[9] H.-T. Yang, J.-R. Huang, and T.-Y. Chang, "A chaos-based fully digital $120 \mathrm{MHz}$ pseudo random number generator," in The 2004 IEEE AsiaPacific Conference on Circuits and Systems., vol. 1, December 2004, pp. 357-360.

[10] A. S. Mansingka, A. G. Radwan, M. A. Zidan, and K. N. Salama, "Analysis of bus width and delay on a fully digital signum nonlinearity chaotic oscillator," in IEEE International Midwest Symposium on Circuits and Systems (MWSCAS'11), August 2011.

[11] A. S. Mansingka, A. G. Radwan, and K. N. Salama, "Design, implementation and analysis of fully digital 1-d controllable multiscroll chaos," in IEEE International Conference on Microelectronics (ICM'11), December 2011.

[12] E. Lorenz, "Deterministic nonperiodic flow," Atmos. Sci, vol. 20, pp. 130-141, 1963.

[13] G. R. Chen and J. H. Lu, "Dynamics of the lorenz system family: Analysis, control and synchronization," Beijing: Science Press, 2003, (in Chinese).

[14] M. Perc, "User friendly programs for nonlinear time series analysis," 2010, [Retrieved 7 August, 2010]. [Online]. Available: http://www. matjazperc.com/ejp/time.html 